\title{
Heterogeneous molecular mechanisms underlie attenuated familial adenomatous polyposis
}

Francesca Cattaneo, $P h D^{1 *}$, Sara Molatore, $P h D^{1 *}$, Markos Mihalatos, $P h D^{2}$, Angela Apessos, $P h D^{2}$, Tiziana Venesio, $\mathrm{PhD}^{3}$, Silvia Bione, $\mathrm{PhD}^{4}$, Pierangela Grignani, $P h D^{5}$, Georgios Nasioulas, $P h D^{2}$, and Guglielmina Nadia Ranzani, $P h D^{1}$

\begin{abstract}
Purpose: Familial adenomatous polyposis is a phenotypically heterogeneous disease predisposing to colorectal cancer. It is dominantly transmitted, when associated with the APC gene, and recessively inherited, when associated with MUTYH gene. We searched for APC and MUTYH germline alterations in Italian and Greek patients with attenuated polyposis, a phenotypic variant whose genetic cause remains unknown in many cases. Methods: We studied 26 unrelated patients (and 16 relatives) with multiple colorectal adenomas (3-100, by endoscopic analysis) that had screened $A P C$ mutation-negative by protein truncation test. We searched for APC rearrangements by multiplex ligationdependent probe amplification and for MUTYH mutations by sequencing. We performed a screening of five MUTYH recurrent pathogenic mutations in 501 Italian and 144 Greek controls. Results: One patient proved to carry an APC whole-gene deletion; 4 of 25 (16\%) patients showed biallelic and 3 of 25 (12\%) monoallelic MUTYH mutations. In the three heterozygous subjects no pathogenetic variants were found in OGG1, MTH1, APE1, MSH2, and MSH6 genes. Frequency assessment of MUTYH mutations in healthy subjects showed that only $\mathrm{Y} 165 \mathrm{C}$ and G382D reach a subpolymorphic frequency. Conclusion: Attenuated polyposis patients without "conventional" APC mutations are genetically heterogeneous, and the phenotype is not directly related to the germline defect. Therefore, the families' appropriate management requires an accurate genetic and clinical investigation. Genet Med 2007:9(12):836-841.
\end{abstract}

Key Words: polyposis, colorectal cancer, phenotype, germline mutations, molecular mechanisms

Familial adenomatous polyposis (FAP) is an inherited syndrome conferring a very high risk of colorectal cancer through the formation of multiple colorectal adenomas. The number of adenomas, the age of onset of adenomatosis and carcinoma, and the possible presence of extracolonic manifestations, vary both between and within the affected families. In the "classic" disease, the number of adenomas ranges from a hundred to thousands and polyps usually emerge during the second-third decade of life. ${ }^{1}$ On the other hand, the so-called "attenuated" polyposis ( $<100$ adenomas) is characterized by a milder course of the disease, a later onset of adenomatosis, and a reduced expression of the extracolonic manifestations; the average age of colon cancer is $50-55$ years, which is about $10-15$ years later than the age found for classic disease. ${ }^{2}$ Although

From the ${ }^{1}$ Department of Genetics and Microbiology, University of Pavia, Pavia, Italy; ${ }^{2}$ Molecular Biology Research Center HYGEIA-Antonis Papayiannis, Athens, Greece; ${ }^{3}$ Unit of Pathology, Institute for Cancer Research and Treatment, Candiolo-Torino; ${ }^{4}$ Institute of Molecular Genetics-CNR; and ${ }^{5}$ Department of Legal Medicine and Public Health, University of Pavia, Pavia, Italy.

Guglielmina Nadia Ranzani, PhD, Department of Genetics and Microbiology, University of Pavia, Via Abbiategrasso 207, 27100 Pavia, Italy.E-mail: ranzani@ipvgen.unipv.it.

${ }^{*}$ Francesca Cattaneo, PhD, and Sara Molatore, PhD contributed equally to this paper.

Disclosure: The authors declare no conflict of interest.

Submitted for publication June 15, 2007.

Accepted for publication August 22, 2007.

DOI: 10.1097/GIM.0b013e31815bf940 diagnostic criteria are consistent with the above characteristics, attenuated polyposis is not a well-defined entity and can either mimic the typical disease or resemble sporadic adenomas and colorectal cancer.

Both classic and attenuated polyposis can be associated with germline mutations of either $A P C$ tumor suppressor gene ${ }^{3,4}$ or MUTYH base excision repair gene. ${ }^{5,6}$ Classic (FAP) and attenuated (AFAP) polyposis linked to APC gene follow a dominant mode of inheritance, whereas the $M U T Y H$-associated disease (MAP) is recessively transmitted. The great majority of APC pathogenetic mutations are base changes leading to a truncated protein product; accordingly, the protein truncation test (PTT) is the common choice as mutation detection method. ${ }^{7}$ PTT, as well as other polymerase chain reaction (PCR)-based methods commonly applied for exon-screening, allows mutation detection in about $70 \%$ of FAP and in only $10 \%$ of AFAP patients. These methods are per se inadequate to detect alterations such as large genomic rearrangements and gene expression defects. Very recently, by means of PTT as well as of other molecular methods, Nielsen et al. ${ }^{8}$ identified APC alterations in $36 \%$ of AFAP families. By using a specific quantitative assay, Sieber et al. ${ }^{9}$ demonstrated that up to $12 \%$ of FAP patients with no apparent $A P C$ germline mutation, were carriers of a gene deletion. Haploinsufficiency because of allelic deletion, ${ }^{9-11} \mathrm{ab}-$ normal ratio of APC isoforms, ${ }^{12}$ and reduced or absent mRNA expression from one $A P C$ allele, ${ }^{11}$ have been shown to be rela- 
tively frequent in FAP patients who had proven APC mutation-negative by conventional methods.

From $5 \%$ to $25 \%$ of $A P C$ mutation-negative cases ${ }^{6,13}$ can be attributed to MUTYH biallelic mutations. All reported mutations have been identified by exon-screening conventional methods that are, so far, the only ones utilized for MUTYH genetic testing. Although biallelic mutations appear more frequent in patients with a milder disease, ${ }^{6,14}$ MUTYH inactivation can result in either classic or attenuated phenotype. ${ }^{8}$ Very recently, Aretz et al. ${ }^{15}$ found that MUTYH biallelic mutations were associated with attenuated or "atypical" phenotype in the great majority of polyposis patients analyzed. Biallelic mutations have also been detected in individuals with early onset colorectal cancer, without polyposis. ${ }^{16,17}$

Genotype-phenotype correlations appear complex in polyposis. The position of mutations along the APC gene correlates with the clinical features, but only to a limited extent. The majority of APC mutations occur in the $5^{\prime}$ half of its coding region. Mutations associated with FAP appear scattered all over the gene, with different hot spots in exon 15. On the contrary, mutations located in the $5^{\prime}$ and $3^{\prime}$ end of the gene, as well as in the alternatively spliced exon 9 , are hypomorphic and mainly result in AFAP..$^{2,18,19}$ MAP patients who are carriers of the same MUTYH biallelic mutations can show either the classic or the attenuated phenotype. ${ }^{6,16}$ On the whole, observations on FAP/AFAP and MAP patients are consistent with the idea that the combined effect of unknown modifier genes, genetic background, and environmental factors, can contribute to the phenotypic manifestations in disease-causing mutation carriers.

We searched for germline alterations in 26 unrelated patients and available relatives, of Italian and Greek origins: all patients were diagnosed with attenuated polyposis (3-100 adenomas) and had proven to be $A P C$ mutation-negative by conventional methods. We searched for both $A P C$ rearrangements and MUTYH mutations. Moreover, we deeply investigated three patients who turned out to carry a single MUTYH mutation, by searching for germline variants in other DNA repair genes. Finally, to improve screening strategy, we evaluated the population frequency of the MUTYH pathogenetic variants that seem to be recurrent in southern Europe patients. ${ }^{14,20}$

\section{PATIENTS AND METHODS}

\section{Samples}

This study was performed on 26 unrelated patients, 20 of Italian and 6 of Greek origin, and on 16 patients' relatives. Greek cases are the last ones in Table 1 (patients 21-26). Italian patients were new cases recruited at the unit of Gastroenterology II, S. Giovanni A.S. Hospital-Torino, from 2002 to 2006; to the best of our knowledge, no proband/affected relative was included in any Italian polyposis registry. The Greek patients and their families were referred through the Gastroenterology Department of Hygeia Hospital, Athens and the Hellenic group for the study of hereditary colorectal cancer during the past 6 years. Clinical and pathologic features were obtained at diagnosis and family history was ascertained by genetic counseling. Patients included in this study were diagnosed with attenuated polyposis ( $<100$ adenomas) on the basis of endoscopic analysis. Most definitions of attenuated polyposis only include subjects with at least 10 polyps. By also considering the age, we selected patients as follows: at least 3 polyps after the age of 30 years plus family history of polyposis and/or colorectal cancer (no family members with "classic" polyposis); at least 6 polyps before the age of 50 years or more than 6 polyps after the age of 50 years, in the absence of family history. Blood samples were obtained after informed consent. DNA and RNA were extracted from peripheral blood by standard procedures and by the QIAmp RNA Blood Mini Kit (Qiagen, Valencia, CA), respectively. All patients of this study were proven to be $A P C$-mutation-negative by PTT and/or DNA sequencing methods. We screened for both $A P C$ rearrangements and MUTYH mutations in patients, by using multiplex ligation-dependent probe amplification (MLPA) and DNA sequencing methods, respectively.

The population screening of MUTYH pathogenetic variants was performed on a control sample including 501 Italian (205 from northern and 296 from southern Italy) and 144 Greek (from Cyclades) healthy subjects with no obvious family history of cancer. Genomic DNA extracted from peripheral blood was used for restriction enzyme digestion.

\section{Search for APC large genomic rearrangements by MLPA}

MLPA $^{21}$ was carried out by using the SALSA P043 APC MLPA kit from MRC-Holland (Amsterdam, The Netherlands), according to the manufacturer's protocols. The kit includes 23 probes for each exon and for the promoter region of the APC gene, as well as 13 probes for other control target DNA sequences throughout the genome. The method is based on: (a) the hybridization of probes to specific genomic DNA sequences; (b) the amplification of the hybridized probe (one primer being fluorescently labeled); (c) the semiquantitative analysis of the PCR products. Labeled PCR products of MLPA were run on the ABI Prism 310 sequencer (Applied Biosystems, Foster City, CA) and analyzed by GenScan Analysis Software Version 3.1.2 (Applied Biosystems); peak height for each fragment was reported to an Excel spreadsheet (available at the website http://www. ngrl.org.uk/Manchester/Publications.htm\#MLPA) designed to assess the ratios of each test peak relative to all other peaks for that individual and the normal controls. A dosage quotient of 1.0 is expected for normal sequences; the dosage quotient should be 0.5 in the presence of a sequence deletion. Each patient was analyzed by two independent MLPA experiments.

\section{MUTYH gene sequencing}

Exons 1-16 of MUTYH were amplified according to AlTassan et al. ${ }^{5}$ and Jones et al. ${ }^{22}$; PCR products were sequenced as previously reported. ${ }^{14}$

\section{Sequencing of OGG1, MTH1, APE1, MSH2, and MSH6 genes}

OGG1 and MTH1 exons were amplified according to Al-Tassan et al. ${ }^{5}$ and Jones et al. ${ }^{22}$ MSH2 and MSH6 exons were amplified according to Holinski-Feder et al. ${ }^{23}$ and Plaschke et al., ${ }^{24}$ respec- 
Table 1

Clinicopathological features and germline alterations of APC-mutation-negative (PTT) patients with multiple colorectal adenomas

\begin{tabular}{|c|c|c|c|c|c|c|c|}
\hline Patient & Sex & $\begin{array}{c}\text { Age at } \\
\text { diagnosis }\end{array}$ & No. polyps & $\begin{array}{l}\text { Colon } \\
\text { cancer }(\mathrm{yr})\end{array}$ & $\begin{array}{l}\text { Family } \\
\text { history }\end{array}$ & MUTYH mutations & MUTYH polymorphic variant \\
\hline 1 & $\mathrm{~F}$ & 35 & $30-40$ & - & None & - & - \\
\hline 2 & M & 54 & 4 & - & $\mathrm{AD}$ & - & - \\
\hline 3 & $\mathrm{~F}$ & 62 & 3 & - & $\mathrm{AD}$ & - & - \\
\hline 4 & M & 61 & $20-30$ & - & None & - & $\mathrm{Q} 324 \mathrm{H}[\mathrm{c} .972 \mathrm{G} \rightarrow \mathrm{C}, \mathrm{p} . \mathrm{Gln} 324 \mathrm{His}]+[=]$ \\
\hline 5 & $\mathrm{~F}$ & 35 & $70-100$ & - & None & - & $\mathrm{Q} 324 \mathrm{H}[\mathrm{c} .972 \mathrm{G} \rightarrow \mathrm{C}, \mathrm{p} . \mathrm{Gln} 324 \mathrm{His}]+[=]$ \\
\hline 6 & M & 60 & 8 & - & None & Y165C [c.494A $\rightarrow$ G, p.Tyr165Cys $]+[=]$ & - \\
\hline 7 & $\mathrm{~F}$ & 56 & $90-100$ & $+(56)$ & $\mathrm{AR}$ & $\begin{array}{l}\text { Y165C/R168C [c.494A } \rightarrow \text { G, p.Tyr165Cys }]+ \\
\quad[\text { c.502C } \rightarrow \text { T, p.Arg168Cys }]\end{array}$ & - \\
\hline 8 & $\mathrm{~F}$ & 42 & 30 & - & None & - & - \\
\hline 9 & M & 39 & 50 & - & None & - & $\begin{array}{l}\text { Q324H/Q324H [c.972G } \rightarrow \text { C, p.Gln324His }]+ \\
\quad[\text { c. } 972 \mathrm{G} \rightarrow \text { C, p.Gln324His }]\end{array}$ \\
\hline 10 & M & 35 & 30 & - & None & $\begin{array}{l}\text { Y90X/W160X }[\text { c.270C } \rightarrow \text { A, p.Tyr90X }]+ \\
\quad[\text { c.479G } \rightarrow \text { A, p.Trp160X] }\end{array}$ & - \\
\hline 11 & $\mathrm{~F}$ & 57 & 6 & - & $\mathrm{AD}$ & - & $\mathrm{Q} 324 \mathrm{H}[\mathrm{c} .972 \mathrm{G} \rightarrow \mathrm{C}, \mathrm{p} . \mathrm{Gln} 324 \mathrm{His}]+[=]$ \\
\hline 12 & M & 46 & 50 & - & None & - & - \\
\hline 13 & M & 73 & $30-40$ & - & $\mathrm{AR}$ & - & $\mathrm{Q} 324 \mathrm{H}[\mathrm{c} .972 \mathrm{G} \rightarrow \mathrm{C}, \mathrm{p} . \mathrm{Gln} 324 \mathrm{His}]+[=]$ \\
\hline 14 & M & 68 & 4 & $+(64)$ & $\mathrm{AD}$ & - & $\mathrm{Q} 324 \mathrm{H}[\mathrm{c} .972 \mathrm{G} \rightarrow \mathrm{C}, \mathrm{p} . \mathrm{Gln} 324 \mathrm{His}]+[=]$ \\
\hline 15 & M & 55 & 6 & - & $\mathrm{AD}$ & - & - \\
\hline 16 & M & 42 & 40 & $+(40,42)$ & None & - & - \\
\hline $17^{a}$ & $\mathrm{~F}$ & 38 & $70-100$ & - & $\mathrm{AD}$ & - & - \\
\hline 18 & $\mathrm{~F}$ & 39 & 7 & $+(39)$ & None & $\begin{array}{l}\text { G382D/G382D [c.1145G } \rightarrow \text { A, p.Gly382Asp }]+ \\
\quad[\text { c.1145G } \rightarrow \text { A, p.Gly382Asp] }\end{array}$ & - \\
\hline 19 & M & 54 & $<40$ & $+(54)$ & None & - & - \\
\hline 20 & $\mathrm{~F}$ & 43 & 6 & - & None & - & $\mathrm{Q} 324 \mathrm{H}[\mathrm{c} .972 \mathrm{G} \rightarrow \mathrm{C}, \mathrm{p} . \mathrm{Gln} 324 \mathrm{His}]+[=]$ \\
\hline 21 & M & 35 & $<100$ & $+(35)$ & $\mathrm{AR}$ & $\begin{array}{l}\text { Q324X/G382D [c.970C } \rightarrow \text { T, p.Gln324X] + } \\
\quad[\text { c.1145G } \rightarrow \text { A, p.Gly382Asp }]\end{array}$ & - \\
\hline 22 & $\mathrm{~F}$ & 23 & 25 & - & None & - & - \\
\hline 23 & $\mathrm{~F}$ & 35 & $10-20$ & - & $\mathrm{AR}$ & - & $\mathrm{Q} 324 \mathrm{H}[\mathrm{c} .972 \mathrm{G} \rightarrow \mathrm{C}, \mathrm{p} . \mathrm{Gln} 324 \mathrm{His}]+[=]$ \\
\hline 24 & M & 66 & $20-30$ & - & None & - & - \\
\hline 25 & M & 48 & $90-100$ & $+(48)$ & $\mathrm{AD}$ & Y165C [c.494A $\rightarrow$ G, p.Tyr165Cys $]+[=]$ & Q324H $[$ c.972G $\rightarrow$ C, p.Gln324His $]+[=]$ \\
\hline 26 & M & 49 & $90-100$ & $+(49)$ & None & $\mathrm{R} 171 \mathrm{~W}[\mathrm{c} .511 \mathrm{C} \rightarrow \mathrm{T}, \mathrm{pArg} 171 \mathrm{rp}]+[=]$ & - \\
\hline
\end{tabular}

Autosomal dominant, $\mathrm{AD}$; autosomal recessive, $\mathrm{AR}$.

${ }^{a} A P C$ locus deletion carrier.

tively. APE1 was analyzed by using primers we specifically designed to amplify exons $1-4$ (primers available on request). All PCR products were sequenced as previously reported. ${ }^{14}$

\section{Analysis of MUTYH and MTH1 variants by restriction enzyme digestion}

We screened for MUTYH pathogenetic variants in cancerfree controls by digesting with the appropriate enzyme the PCR product corresponding to the exon of interest. In particular, missense mutation Y165C (exon 7) was analyzed by BseXI, frameshift mutation 1103delC (exon 12) by BglI, G382D missense and 1187insGG frameshift mutations (exon
13) by BglII, and 1395delGGA (exon 14) in frame deletion by MnlI restriction enzymes. The frequency of the R171W (exon 7) missense variant was assessed by means of SsiI digestion. Finally, the silent substitution in exon 4 of MTH1 gene was investigated by Eam1105I enzyme. After digestion, restriction fragments were separated by electrophoresis on acrylamide gel at the appropriate concentration.

\section{RESULTS}

By MLPA approach, we investigated 26 patients who had tested APC-mutation-negative by conventional methods: 1 of 
26 proved to carry an $A P C$ gene deletion. In this subject, three independent MLPA experiments demonstrated a reduction of all $A P C$-specific peaks compared with control peaks, suggesting the presence of a deletion encompassing the entire APC locus. The whole-gene deletion carrier had a dominant family history of polyposis and showed a "milder" phenotype with $70-100$ adenomas at 38 years of age (patient 17 of Table 1).

We analyzed the MUTYH coding sequence (16 exons and intron/exon boundaries) in the remaining 25 patients. We found four subjects with MUTYH biallelic mutations (patients 7, 10, 18, 21 of Table 1): all mutations, already found in other polyposis patients, were nonsense or missense mutations predicted to impair the MUTYH protein function severely. Biallelic mutation carriers showed a variable number of polyps (from 7 to about 100); three of four subjects had developed a colorectal cancer at young age (patients 7, 18, 21 of Table 1). We performed MUTYH mutation analysis on two children of proband (patient 7); we also analyzed 13 relatives of proband (patient 21), including 2 affected sisters and 11 nephews: the results were consistent with a recessive transmission of the disease. The affected sisters of proband (patient 21) showed his same mutations, but a milder phenotype, with $20-30$ polyps and no colorectal cancer at 54 and 57 years of age, respectively.

DNA sequencing revealed a single MUTYH mutation in three probands, two of which showing about 100 polyps and colorectal cancer at young age (patients 6, 25, 26 of Table 1). Patients 6 and 25 were carriers of the Y165C recurrent pathogenetic mutation, whereas patient 26 showed a novel mutation with likely functional significance (C to $\mathrm{T}$ at nucleotide 511; $\mathrm{R} 171 \mathrm{~W})$. Indeed, the amino acid at position 171 is localized within the catalytic domain of MUTYH protein and it is evolutionary conserved across species (http://www.ebi.ac.uk/ clustalw/index.html). This mutation must be rare in the population because the restriction analysis we carried out on 100 healthy controls did not reveal any mutation carrier. Patients 6 and 26 had no family history. On the contrary, patient 25 had an apparently dominant history of colorectal cancer. His father and his paternal aunt died of colorectal cancer late in life, at 72 and 78 years of age, respectively; his brother developed $<100$ polyps and a colorectal cancer at 61 years of age. The analysis of the affected brother showed the presence of the Y165C mutated allele.

We investigated the three MUTYH heterozygous probands for the presence of germline mutations in DNA repair genes other than MUTYH, namely OGG1, MTH1, APE1, MSH2, and MSH6. The direct sequencing of the entire coding region of these genes did not reveal any alteration, with one exception. In patient 25 and in his affected brother, both carrying the Y165C pathogenetic variant, we detected a mutation of the MTH1 gene ( $\mathrm{C}$ to $\mathrm{T}$ at nucleotide 366 ). This mutation consisted of a silent nucleotide substitution at the second-last base of exon 4 (rs35932242 in the National Center for Biotechnology Information Single Nucleotide Polymorphism [NCBI SNP] database). According to in silico analysis, this change is not predicted to affect the splicing. Restriction analysis we carried out on 100 cancer-free controls revealed three heterozygous carri- ers of the MTH1 mutation (frequency: 1.5\%). Taken together, these observations make it extremely unlikely that polyposis could be caused by the MTH1 variant in "compound heterozygosity" with the Y165C MUTYH pathogenetic mutation.

As far as MUTYH known polymorphisms are concerned, no patient showed the V22M variant, whereas nine patients (eight heterozygotes and one homozygote; Table 1) proved to carry the Q324H common allele (rs3219484 and rs3219489 in the NCBI SNP database, respectively).

To possibly improve the strategy of pathogenetic mutation screening, we evaluated the frequency of the MUTYH variants that are recurrent in southern Europe patients. By means of restriction enzymatic digestion, we screened for Y165C, 1103delC, G382D, 1187insGG, and 1395delGGA variants in 645 healthy subjects including 501 Italians ( 205 from northern and 296 from southern Italy) and 144 Greeks (Cyclades). No variants were found in the Greek sample. The G382D mutation was found in two individuals from northern and in one individual from southern Italy. Both Y165C and 1187insGG variants were found in a single subject from northern Italy. All mutation carriers were heterozygous.

\section{DISCUSSION}

We focused our attention on attenuated FAP, a disease phenotypic variant whose genetic cause remains unknown in up to $30-80 \%$ of cases.

To identify rearrangements such as deletions/amplifications of either a part or the entire APC locus, we applied the MLPA method to 26 patients that screened $A P C$ mutation-negative by common techniques. One subject proved to carry a wholegene deletion. Various investigations failed to detect wholegene deletions in patients with attenuated polyposis, and demonstrated that this genetic lesion may occur with a frequency higher than $10 \%$ in mutation-negative subjects with the typical disease. ${ }^{9,11,25}$ However, Pilarski et al. ${ }^{26}$ reported an attenuated polyposis case associated with the germline deletion of the entire APC gene. Su et al. ${ }^{27}$ characterized two deletions of the entire APC exon 15: one proband had the typical phenotype, whereas the other one had a phenotype consistent with attenuated polyposis. Very recently, Nielsen et al. ${ }^{28}$ reported two families carrying a deletion of exons $7-13$ and showing an attenuated phenotype.

Our deletion carrier was a 38-year-old woman with $<100$ polyps and no other clinical manifestations. These observations indicate that, although rarely, APC haploinsufficiency because of allelic deletion can result in an attenuated/mild phenotype.

Sieber et al. ${ }^{6}$ reported that about $5 \%$ of patients with attenuated polyposis had disease attributable to $M U T Y H$, whereas among patients with more severe disease (15-100 adenomas) mutation carriers were $30 \%$. Among PTT-negative patients with $\leq 100$ adenomas, we found $16 \%$ (4 of 25) carriers of MUTYH biallelic mutations. However, if we only consider patients with more than 30 adenomas and no vertical transmission of the disease, the frequency of MUTYH carriers reaches 
$25 \%$ ( 3 of 12) of value. By applying these last criteria to a different series of attenuated polyposis patients, we previously found a particularly high frequency of biallelic mutation carriers (60\%). ${ }^{14}$ This difference may depend on the small number of patients included in both previous and present surveys. However, our data, as well as data from other authors, clearly indicate that, although MAP patients can show different phenotypes, germline mutations of MUTYH gene are frequently associated with a milder phenotype.

Three probands, two of which were affected with colorectal cancer, were found to be heterozygous carriers of pathogenetic mutations. To verify the occurrence of other possible pathogenetic mutations in these patients, we analyzed OGG1, MTH1, $A P E 1, M S H 6$, and $M S H 2$ genes. These genes have a role in base excision repair and/or in mismatch repair pathways and their products can interact with each other mutually. No mutations clearly affecting the protein functions could be identified. Therefore, either a different gene from those we investigated is responsible for attenuated polyposis (either per se or in association with a single MUTYH functional mutation), or a single MUTYH functional mutation, in association with modifier genes, genetic background, and/or environmental factors, can give rise to the disease. In addition, we cannot exclude that genetic alterations undetectable by sequencing, such as expression or splicing defects, may affect the MUTYH "wild-type" allele in these patients.

The presence of single MUTYH pathogenetic mutations in polyposis patients has been reported by different authors. ${ }^{6,13}$ Apparently, such a heterozygous status does not correlate with the severity of polyposis phenotype (see Refs. 6 and 13; present data). Sieber et al. ${ }^{6}$ reported that $A P C$ somatic mutations in polyps from heterozygous patients were not $\mathrm{G}: \mathrm{C} \rightarrow \mathrm{T}: \mathrm{A}$ transversions that are typically caused by MUTYH deficiency. However, at present it is not possible to exclude a mechanism that in some circumstances might allow germline partial-deficiency/ haploinsufficiency of MUTYH gene to have some role in polyposis development.

Intriguingly, heterozygous mutations have been reported to increase the risk of colorectal cancer without polyposis later in life. ${ }^{17}$ Moreover, both biallelic and monoallelic mutation carriers are more likely to have first and/or second-degree relatives with colorectal cancer compared with noncarriers. ${ }^{29}$ These observations, as well as those reported on colon cancer families who lack mismatch repair gene defects, ${ }^{30}$ suggest that MUTYH heterozygous mutations represent low-penetrance colorectal cancer-causing alleles. On the other hand, recently performed meta-analyses produced conflicting results, indicating both a nonsignificant relative risk of colorectal cancer $^{31,32}$ and a small but significantly increased risk of cancer ${ }^{33}$ for monoallelic mutation carriers.

An apparently dominant MAP has been reported for some families. ${ }^{34,35}$ This finding might be explained by the frequency of heterozygotes in the general population: in a fraction of cases, a carrier of two MUTYH mutations is expected to have children with a partner who carries a single mutation. In addi- tion, the disease might be associated with MUTYH monoallelic mutations in relation with modifier genes (see Ref. 6; patient 25 of Table 1). Therefore, MUTYH genetic testing has to be considered also for $A P C$ mutation-negative families with vertical transmission of the disease.

In our sample, cancer was present in three of four patients with MUTYH biallelic mutations, in two of three with monoallelic mutations, and only in 2 of the 18 patients without mutations. This observation seems to emphasize the high risk of malignant degeneration in MUTYH mutation carriers and raises the question of an appropriate surveillance (for a critical discussion see Ref. 35).

To possibly improve the MUTYH screening strategy in patients from southern Europe, we assessed the frequency of some recurrent pathogenetic variants in the normal population. Y165C and G382D mutations (also predominant in northern Europe) showed subpolymorphic frequencies, whereas the other selected mutations proved to be rare. This observation and the finding that different MUTYH pathogenetic mutations have been reported in polyposis patients from southern Europe (see Refs. 14, 20, and 36; present data), make it difficult to follow a predetermined strategy. Therefore, although starting from Y165C and G382D mutations, the analysis of the entire MUTYH gene is highly recommended for genetic testing.

In addition to the phenotypic heterogeneity of both classical and attenuated polyposis, ${ }^{19}$ the analysis of extracolonic manifestations and the number of colon adenomas depend on different factors, including the expertise and knowledge of the gastroenterologist and the quality of the endoscopy. Accordingly, Lynch and Smyrk ${ }^{37}$ defined the disease "a diagnostic nightmare." It has been pointed out that disease severity in attenuated polyposis varies greatly but it depends on the site of APC germline mutation. ${ }^{19}$ However, germline mutations can affect either $A P C$ or $M U T Y H$ genes. In turn, not only truncating mutations in the $5^{\prime}, 3^{\prime}$, and exon 9 of the $A P C$ gene can give rise to the attenuated phenotype, but full/partial APC gene deletions too. Moreover, different functional biallelic mutations of MUTYH can account for a fraction of patients. Finally, MUTYH single mutations are also suspected to play a role in the disease development.

\section{ACKNOWLEDGMENTS}

This research was supported by PRIN-MIUR (Ministero dell'Istruzione, dell'Università e della Ricerca, Italy); Fondazione Cariplo (Nobel); Hygeia S.A. Hospital.

\section{References}

1. Vasen HF. Clinical diagnosis and management of hereditary colorectal cancer syndromes. J Clin Oncol 2000;18:81S-92S.

2. Knudsen AL, Bisgaard ML, Bulow S. Attenuated familial adenomatous polyposis (AFAP). A review of the literature. Fam Cancer 2003;2:43-55.

3. Groden J, Thliveris A, Samowitz W, Carlson M, et al. Identification and characterization of the familial adenomatous polyposis coli gene. Cell 1991;66:589-600.

4. Kinzler KW, Nilbert MC, Su LK, Vogelstein B, et al. Identification of FAP locus genes from chromosome 5q21. Science 1991;253:661-665.

5. Al-Tassan N, Chmiel NH, Maynard J, Fleming N, et al. Inherited variants of $M Y H$ associated with somatic G:C $\rightarrow$ T:A mutations in colorectal tumors. Nat Genet 2002; $30: 227-232$. 
6. Sieber OM, Lipton L, Crabtree M, Heinimann K, et al. Multiple colorectal adenomas, classic adenomatous polyposis, and germ-line mutations in MYH. N Engl J Med 2003;348:791-799.

7. Powell SM, Petersen GM, Krush AJ, Booker S, et al. Molecular diagnosis of familial adenomatous polyposis. N Engl J Med 1993;329:1982-1987.

8. Nielsen M, Hes FJ, Nagengast FM, Weiss MM, et al. Germline mutations in APC and MUTYH are responsible for the majority of families with attenuated familial adenomatous polyposis. Clin Genet 2007;71:427-433.

9. Sieber OM, Lamlum H, Crabtree MD, Rowan AJ, et al. Whole-gene APC deletions cause classical familial adenomatous polyposis, but not attenuated polyposis or "multiple" colorectal adenomas. Proc Natl Acad Sci USA 2002;99:2954-2958.

10. Venesio T, Balsamo A, Rondo-Spaudo M, Varesco L, et al. APC haploinsufficiency, but not $C T N N B 1$ or $C D H 1$ gene mutations, accounts for a fraction of familial adenomatous polyposis patients without APC truncating mutations. Lab Invest 2003;83: 1859-1866.

11. Renkonen ET, Nieminen P, Abdel-Rahman WM, Moisio AL, et al. Adenomatous polyposis families that screen $A P C$ mutation-negative by conventional methods are genetically heterogeneous. J Clin Oncol 2005;23:5651-5659.

12. Venesio T, Balsamo A, Sfiligoi C, Fuso L, et al. Constitutional high expression of an APC mRNA isoform in a subset of attenuated familial adenomatous polyposis patients. J Mol Med 2007;85:301-308.

13. Nielsen M, Franken PF, Reinards TH, Weiss MM, et al. Multiplicity in polyp count and extracolonic manifestations in 40 Dutch patients with $M Y H$ associated polyposis coli (MAP). J Med Genet 2005;42:e54.

14. Venesio T, Molatore S, Cattaneo F, Arrigoni A, et al. High frequency of MYH gene mutations in a subset of patients with familial adenomatous polyposis. Gastroenterology 2004;126:1681-1685.

15. Aretz S, Uhlhaas S, Goergens H, Siberg K, et al. MUTYH-associated polyposis: 70 of 71 patients with biallelic mutations present with an attenuated or atypical phenotype. Int J Cancer 2006;119:807-814.

16. Wang L, Baudhuin LM, Boardman LA, Steenblock KJ, et al. MYH mutations in patients with attenuated and classic polyposis and with young-onset colorectal cancer without polyps. Gastroenterology 2004;127:9-16.

17. Farrington SM, Tenesa A, Barnetson R, Wiltshire A, et al. Germline susceptibility to colorectal cancer due to base-excision repair gene defects. Am J Hum Genet 2005;77: $112-119$.

18. de la Chapelle A. Genetic predisposition to colorectal cancer. Nat Rev Cancer 2004;4: $769-780$.

19. Sieber OM, Segditsas S, Knudsen AL, Zhang J, et al. Disease severity and genetic pathways in attenuated familial adenomatous polyposis vary greatly but depend on the site of the germline mutation. Gut 2006;55:1440-1448.

20. Gismondi V, Meta M, Bonelli L, Radice P, et al. Prevalence of the Y165C, G382D and 1395delGGA germline mutations of the $M Y H$ gene in Italian patients with adenomatous polyposis coli and colorectal adenomas. Int J Cancer 2004;109:680-684.

21. Schouten JP, McElgunn CJ, Waaijer R, Zwijnenburg D, et al. Relative quantification of 40 nucleic acid sequences by multiplex ligation-dependent probe amplification. Nucleic Acids Res 2002;30:e57.

22. Jones S, Emmerson P, Maynard J, Best JM, et al. Biallelic germline mutations in $M Y H$ predispose to multiple colorectal adenoma and somatic $\mathrm{G}: \mathrm{C} \rightarrow \mathrm{T}:$ A mutations. Hum Mol Genet 2002;11:2961-2967.

23. Holinski-Feder E, Muller-Koch Y, Friedl W, Moeslein G, et al. DHPLC mutation analysis of the hereditary nonpolyposis colon cancer (HNPCC) genes $h M L H 1$ and hMSH2. J Biochem Biophys Methods 2001;47:21-32.

24. Plaschke J, Kruppa C, Tischler R, Bocker T, et al. Sequence analysis of the mismatch repair gene $h M S H 6$ in the germline of patients with familial and sporadic colorectal cancer. Int J Cancer 2000;85:606-613.

25. Michils G, Tejpar S, Thoelen R, van Cutsem E, et al. Large deletions of the APC gene in $15 \%$ of mutation-negative patients with classical polyposis (FAP): a Belgian study. Hum Mutat 2005;25:125-134.

26. Pilarski RT, Brothman AR, Benn P, Shulman Rosengren S. Attenuated familial adenomatous polyposis in a man with an interstitial deletion of chromosome arm 5q. Am J Med Genet 1999;86:321-324.

27. Su LK, Kohlmann W, Ward PA, Lynch PM. Different familial adenomatous polyposis phenotypes resulting from deletions of the entire APC exon 15. Hum Genet 2002;111: $88-95$.

28. Nielsen M, Bik E, Hes FJ, Breuning MH, et al. Genotype-phenotype correlations in 19 Dutch cases with APC gene deletions and a literature review. Eur J Hum Genet 2007; 15:1034-1042.

29. Croitoru ME, Cleary SP, Di Nicola N, Manno M, et al. Association between biallelic and monoallelic germline $M Y H$ gene mutations and colorectal cancer risk. J Natl Cancer Inst 2004;96:1631-1634.

30. Peterlongo P, Mitra N, Abajo AS, Hoya Mde L, et al. Increased frequency of diseasecausing MYH mutations in colon cancer families. Carcinogenesis 2006;27:2243-2249.

31. Webb EL, Rudd MF, Houlston RS. Colorectal cancer risk in monoallelic carriers of MYH variants. Am J Hum Genet 2006;79:768-771.

32. Balaguer F, Castellvi-Bel S, Castells A, Andreu M, et al. Identification of MYH mutation carriers in colorectal cancer: a multicenter, case-control, population-based study. Clin Gastroenterol Hepatol 2007;5:379-387.

33. Tenesa A, Campbell H, Barnetson R, Porteous M, et al. Association of MUTYH and colorectal cancer. Br J Cancer 2006;95:239-242.

34. Lejeune S, Guillemot F, Triboulet JP, Cattan S, et al. Low frequency of AXIN2 mutations and high frequency of MUTYH mutations in patients with multiple polyposis. Hum Mutat 2006;27:1064.

35. Leite JS, Isidro G, Martins M, Regateiro F, et al. Is prophylactic colectomy indicated in patients with $M Y H$-associated polyposis? Colorectal Dis 2005;7:327-331.

36. Isidro G, Laranjeira F, Pires A, Leite J, et al. Germline MUTYH (MYH) mutations in Portuguese individuals with multiple colorectal adenomas. Hum Mutat 2004;24:353354.

37. Lynch HT, Smyrk TC. Classification of familial adenomatous polyposis: a diagnostic nightmare. Am J Hum Genet 1998;62:1288-1289. 